Open Access

\title{
Epidemiological associations between brachycephaly and upper respiratory tract disorders in dogs attending veterinary practices in England
}

Dan G. O'Neill ${ }^{1 *}$, Caitlin Jackson², Jonathan H. Guy², David B. Church¹, Paul D. McGreevy ${ }^{3}$, Peter C. Thomson ${ }^{3}$ and Dave C. Brodbelt ${ }^{1}$

\begin{abstract}
Background: Brachycephalic dog breeds are increasingly common. Canine brachycephaly has been associated with upper respiratory tract (URT) disorders but reliable prevalence data remain lacking. Using primary-care veterinary clinical data, this study aimed to report the prevalence and breed-type risk factors for URT disorders in dogs.

Results: The sampling frame included 170,812 dogs attending 96 primary-care veterinary clinics participating within the VetCompass Programme. Two hundred dogs were randomly selected from each of three extreme brachycephalic breed types (Bulldog, French Bulldog and Pug) and three common small-to medium sized breed types (moderate brachycephalic: Yorkshire Terrier and non-brachycephalic: Border Terrier and West Highland White Terrier). Information on all URT disorders recorded was extracted from individual patient records. Disorder prevalence was compared between groups using the chi-squared test or Fisher's test, as appropriate. Risk factor analysis used multivariable logistic regression modelling.

During the study, 83 (6.9\%) study dogs died. Extreme brachycephalic dogs (median longevity: 8.6 years, IQR: 2.4-10.8) were significantly younger at death than the moderate and non-brachycephalic group of dogs (median 12.7 years, IQR 11.1-15.0) $(P<0.001)$. A higher proportion of deaths in extreme brachycephalic breed types were associated with URT disorders (4/24 deaths, $16.7 \%)$ compared with the moderate and non-brachycephalic group (0/59 deaths, $0.0 \%)(P=0.001)$.

The prevalence of having at least one URT disorder in the extreme brachycephalic group was higher (22.0\%, $95 \%$ confidence interval (Cl): 18.0-26.0) than in the moderate and non-brachycephalic group ( $9.7 \%, 95 \% \mathrm{Cl}: 7.1-12.3, P<0.001)$. The prevalence of URT disorders varied significantly by breed type: Bulldogs $19.5 \%$, French Bulldogs $20.0 \%$, Pugs $26.5 \%$, Border Terriers $9.0 \%$, West Highland White Terriers $7.0 \%$ and Yorkshire Terriers $13.0 \%(P<0.001)$. After accounting for the effects of age, bodyweight, sex, neutering and insurance, extreme brachycephalic dogs had 3.5 times (95\% Cl: 2.4-5.0, $P<0.001$ ) the odds of at least one URT disorder compared with the moderate and non-brachycephalic group.

(Continued on next page)
\end{abstract}

\footnotetext{
*Correspondence: doneill@rvc.ac.uk

${ }^{1}$ The Royal Veterinary College, Hawkshead Lane, North Mymms, HatfieldHerts AL9 7TA, UK

Full list of author information is available at the end of the article
} 
(Continued from previous page)

Conclusions: In summary, this study reports that URT disorders are commonly diagnosed in Bulldog, French Bulldog, Pug, Border Terrier, WHWT and Yorkshire Terrier dogs attending primary-care veterinary practices in England. The three extreme brachycephalic breed types (Bulldog, French Bulldog and Pug) were relatively short-lived and predisposed to URT disorders compared with three other small-to-medium size breed types that are commonly owned (moderate brachycephalic Yorkshire Terrier and non-brachycephalic: Border Terrier and WHWT).

Keywords: Extreme brachycephalic, Moderate brachycephalic, Non-brachycephalic, Upper respiratory, Breed type, Dog, primary-care, VetCompass

\section{Lay summary}

The domestic dog has been artificially selected to meet many different needs and desires of mankind. Skull shape, in particular, has been selected for substantial evolution and is a defining feature for many of the 400 breeds of dog that currently exist. Based on head-shape, breeds can categorised as dolichocephalic (long slender skull), mesaticephalic (intermediate skull conformation) or brachycephalic (braincase longer than facial bones). Brachycephalic dog breeds are increasingly common but canine brachycephaly has been associated with increased upper respiratory tract (URT) disorders. Clinical veterinary data have been used to examine the health of dogs.

This study aimed to use veterinary clinical data to compare URT disorders in three extreme brachycephalic dog breeds (Bulldog, French Bulldog and Pug) with three other commonly owned breeds (Yorkshire Terrier (moderate brachycephalic) and Border Terrier and West Highland White Terrier (non-brachycephalic)).

Extreme brachycephalic dogs were significantly younger at death than the moderate and non-brachycephalic group of dogs (8.6 years vs 12.7 years). The proportion of dogs with at least one URT disorder in the extreme brachycephalic group was higher than in the moderate and non-brachycephalic group (22.0\% vs $9.7 \%)$, and also varied between the breeds: Bulldogs $19.5 \%$, French Bulldogs 20.0 \%, Pugs $26.5 \%$, Yorkshire Terriers $13.0 \%$, Border Terriers $9.0 \%$ and West Highland White Terriers $7.0 \%$. Extreme brachycephalic dogs overall were 3.5 times more likely to have at least one URT disorder compared with the moderate and non-brachycephalic group.

This study helps us to understand how common URT disorders are overall and especially in extreme brachycephalic breeds. The results suggest that owners and veterinarians should be more vigilant for URT disorders and also that breeders should select against extreme body conformations in predisposed breeds.

\section{Background}

The modern domestic dog (Canis lupus familiaris) has been selected to meet the needs and desires of mankind [1]. Although the first distinctive breeds appeared
3,000-4,000 years ago [2], an explosion of companion dog-breeding and breeds during the Victorian era [3] has led to over 400 extant breeds with morphological diversity that is unparalleled among other species [4-6]. Skull morphology, in particular, has evolved substantially over recent centuries and is an important criterion in the written standard for many pedigree breeds, with wide variation described between breeds [7, 8]. Based on head-shape conformation, breeds have been categorised as dolichocephalic (long slender skull), mesaticephalic (intermediate skull conformation) or brachycephalic (braincase longer than facial bones) [9], although newer cephalic index systems derived from various ratios of skull width to skull length ratio aim to describe head shape using a continuous scale $[10,11]$.

Brachycephalic breeds of dog, including Bulldogs, French Bulldogs and Pugs, have become increasingly common in recent years $[12,13]$. The current popularity of smaller brachycephalic breeds may result from a neotenic similarity in head shape to human infants [11] and a generally less fearful nature towards strangers compared with dolichocephalic breeds [14]. This shift from functional to aesthetic selection pressure may have introduced a tolerance for skull morphology that can be associated with health problems [8].

Predisposition to upper respiratory tract (URT) disorders including stenotic nares, enlarged tonsils, elongated soft palate, everted lateral saccules of the larynx, narrowed rima glottides, collapse of the larynx and tracheal hypoplasia have been reported in brachycephalic dog breeds $[15,16]$. Individual dogs may have one, or a combination, of such URT conditions which can additionally predispose to other URT disorders and can be variously combined to describe an overall brachycephalic obstructive airway syndrome (BOAS) [17]. However, a pervading acceptance of some URT disorders especially within some brachycephalic breeds as 'normal for breed' may constrain reforms intended to improve the welfare of affected breeds by 'blinding' vets, owners and breeders to the welfare impacts of these disorders $[18,19,16]$. Reliable data on breed-associated risks of URT disorders are essential to underpin breed improvement strategies 
Table 1 Demographic comparison between two breed groups of 600 extreme brachycephalic (Bulldog, French Bulldog, Pug) and 600 moderate and non-brachycephalic dogs (moderate: Yorkshire Terrier, non-brachycephalic: Border Terrier and West Highland White Terrier) attending primary-care veterinary practices in England (200 dogs of each breed type)

\begin{tabular}{|c|c|c|c|c|c|}
\hline Variable & Category & $\begin{array}{l}\text { Extreme brachycephalic } \\
\text { No. (\%) }\end{array}$ & $\begin{array}{l}\text { Moderate and non-brachycephalic } \\
\text { No. (\%) }\end{array}$ & Overall & $P$-value \\
\hline \multirow[t]{4}{*}{ Sex/neuter } & Female entire & $236(40.0)$ & $160(26.7)$ & $396(33.3)$ & \multirow[t]{4}{*}{$<0.001$} \\
\hline & $\begin{array}{l}\text { Female } \\
\text { neutered }\end{array}$ & $43(7.3)$ & $115(19.2)$ & $158(13.3)$ & \\
\hline & Male entire & $269(45.6)$ & $211(35.2)$ & $480(40.3)$ & \\
\hline & $\begin{array}{l}\text { Male } \\
\text { neutered }\end{array}$ & $42(7.1)$ & $114(19.0)$ & $156(13.1)$ & \\
\hline \multirow[t]{2}{*}{ Insured } & Insured & $103(17.2)$ & $159(26.5)$ & $262(21.8)$ & \multirow[t]{2}{*}{$<0.001$} \\
\hline & Not insured & $497(82.8)$ & $441(73.5)$ & $938(78.2)$ & \\
\hline Bodyweight (kg): Median (IQR ${ }^{\mathrm{a}}$, range) & & $12.4(9.5-24.2,1.6-41.0)$ & $8.7(5.9-10.5,1.5-20.7)$ & $\begin{array}{l}9.7(7.6-12.2,1.5- \\
41.0)\end{array}$ & $<0.001$ \\
\hline $\begin{array}{l}\text { Age (years) at final } E P R^{b} \text { : Median } \\
\left({ }^{Q} R^{\mathrm{a}} \text {, range) }\right.\end{array}$ & & $1.5(0.4-3.5,0.0-14.0)$ & $5.5(2.5-10.2,0.0-18.2)$ & $\begin{array}{l}3.1(1.0-7.1,0.0- \\
18.2)\end{array}$ & $<0.001$ \\
\hline $\begin{array}{l}\text { Time in study (years): Median (IQR', } \\
\text { range) }\end{array}$ & & $0.2(0.0-1.1,0.0-4.3)$ & $0.9(0.0-2.4,0.0-5.0)$ & $\begin{array}{l}0.4(0.0-1.8,0.0- \\
5.0)\end{array}$ & $<0.001$ \\
\hline
\end{tabular}

IQR interquartile range

${ }^{\mathrm{b}} E P R$ electronic patient record

to select against specific disorders [20]. Primary-care veterinary clinical data have been shown to be useful to generate disorder prevalence data that can be generalised to the wider owned-dog population [21].

This study aimed to report and compare the prevalence of URT disorders diagnosed in three extreme brachycephalic dog breed types; namely the Bulldog, French Bulldog and Pug, with three other commonly owned breed types; namely the Yorkshire Terrier (moderate brachycephalic) and Border Terrier and West Highland White Terrier (non-brachycephalic). Additionally, the study aimed to identify risk factors associated with URT disorder diagnosis in the six breed types evaluated. It was hypothesised that extreme brachycephaly is a strong risk factor for URT

Table 2 Comparative demography between three individual extreme brachycephalic breed types (Bulldog, French Bulldog, Pug) and three other breed types (moderate brachycephalic: Yorkshire Terrier, non-brachycephalic: Border Terrier, West Highland White Terrier) of dogs attending primary-care veterinary practices in England (200 dogs of each breed type)

\begin{tabular}{|c|c|c|c|c|c|c|c|c|}
\hline Variable & Category & $\begin{array}{l}\text { Bulldog No. } \\
(\%)\end{array}$ & $\begin{array}{l}\text { French } \\
\text { bulldog No. } \\
(\%)\end{array}$ & Pug No. (\%) & $\begin{array}{l}\text { Border terrier } \\
\text { No. (\%) }\end{array}$ & $\begin{array}{l}\text { West highland white } \\
\text { terrier No. (\%) }\end{array}$ & $\begin{array}{l}\text { Yorkshire } \\
\text { terrier No. (\%) }\end{array}$ & $\begin{array}{l}P- \\
\text { value }\end{array}$ \\
\hline \multirow[t]{4}{*}{ Sex/neuter } & $\begin{array}{l}\text { Female } \\
\text { entire }\end{array}$ & $80(40.4)$ & $83(42.6)$ & $73(37.1)$ & $52(26.0)$ & $51(25.5)$ & $57(28.5)$ & $<0.001$ \\
\hline & $\begin{array}{l}\text { Female } \\
\text { neutered }\end{array}$ & $15(7.6)$ & $11(5.6)$ & $17(8.6)$ & $36(18.0)$ & $43(21.5)$ & $36(18.0)$ & \\
\hline & $\begin{array}{l}\text { Male } \\
\text { entire }\end{array}$ & $87(43.9)$ & $94(48.2)$ & $88(44.7)$ & $74(37.0)$ & $66(33.0)$ & $71(35.5)$ & \\
\hline & $\begin{array}{l}\text { Male } \\
\text { neutered }\end{array}$ & $16(8.1)$ & $7(3.6)$ & $19(9.6)$ & $38(19.0)$ & $40(20.0)$ & $36(18.0)$ & \\
\hline \multirow[t]{2}{*}{ Insured } & Insured & $49(24.5)$ & $20(10.0)$ & $34(17.0)$ & $53(26.5)$ & $60(30.0)$ & $46(23.0)$ & $<0.001$ \\
\hline & $\begin{array}{l}\text { Not } \\
\text { insured }\end{array}$ & $151(75.5)$ & $180(90.0)$ & $166(83.0)$ & $147(73.5)$ & $140(70.0)$ & $154(77.0)$ & \\
\hline $\begin{array}{l}\text { Bodyweight: Median } \\
\text { (IQRª, range) }\end{array}$ & & $\begin{array}{l}27.7(23.6-31.0 \\
11.4-41.0)\end{array}$ & $\begin{array}{l}12.2(11.0- \\
14.0,1.6-19.0)\end{array}$ & $\begin{array}{l}8.9(8.0-10.2, \\
4.9-14.1)\end{array}$ & $\begin{array}{l}10.0(8.6-11.2 \\
4.0-20.7)\end{array}$ & $\begin{array}{l}9.5(8.3-11.0,5.8- \\
14.2)\end{array}$ & $\begin{array}{l}4.75(3.4-6.0 \\
1.5-11.2)\end{array}$ & $<0.001$ \\
\hline $\begin{array}{l}\text { Age (years) at final } E P R^{b} \text { : } \\
\text { Median (IQR }{ }^{a} \text {, range) }\end{array}$ & & $\begin{array}{l}2.0(0.6-4.2, \\
0.0-14.0)\end{array}$ & $\begin{array}{l}1.2(0.4-2.5 \\
0.0-13.0)\end{array}$ & $\begin{array}{l}2.0(0.4-3.8 \\
0.0-13.0)\end{array}$ & $\begin{array}{l}5.2(2.3-9.1 \\
0.0-18.2)\end{array}$ & $\begin{array}{l}6.9(3.4-10.6,0.2- \\
17.9)\end{array}$ & $\begin{array}{l}5.3(2.0-10.3 \\
0.0-17.0)\end{array}$ & $<0.001$ \\
\hline $\begin{array}{l}\text { Time in study (years): } \\
\text { Median (IQR }{ }^{\mathrm{a}} \text {, range) }\end{array}$ & & $\begin{array}{l}0.2(0.0-1.2 \\
0.0-4.0)\end{array}$ & $\begin{array}{l}0.2(0.0-0.9 \\
0.0-3.2)\end{array}$ & $\begin{array}{l}0.2(0.0-1.2 \\
0.0-4.3)\end{array}$ & $\begin{array}{l}1.0(0.0-2.4 \\
0.0-4.6)\end{array}$ & $1.0(0.0-2.5,0.0-5.0)$ & $\begin{array}{l}0.8(0.0-2.2 \\
0.0-4.6)\end{array}$ & $<0.001$ \\
\hline
\end{tabular}


disorders in dogs. These results can inform an evidencebased approach to quantify the impact of URT disorders on the health of the general population of dogs and to evaluate the role of breed type in causation.

\section{Results}

The study sampling frame comprised 170,812 dogs attending 96 clinics from September 1, 2009 until March 2, 2014, and included 1,416 (0.8 \%) Bulldogs, 863 (0.5 \%) French Bulldogs, 1,503 (0.9 \%) Pugs, 1,939 (1.1 \%) Border Terriers, 4,384 (2.6 \%) West Highland White Terriers and 5,594 (3.3 \%) Yorkshire Terriers. The extreme brachycephalic dogs were significantly more likely to be entire, non-insured, heavier, younger and to contribute less time to the study than the moderate and non-brachycephalic dogs $(P<0.001)$ (Table 1$)$. Insurance uptake varied significantly between the breed types: Bulldog 24.5 \%, French Bulldog 10.0 \%, Pug 17.0 \%, Yorkshire Terrier $23.0 \%$, Border Terrier $26.5 \%$ and West Highland White Terrier $30.0 \%(P<0.001)$. Median breed type bodyweight varied significantly, from the Bulldog $(27.7 \mathrm{~kg})$ to the Yorkshire Terrier $(4.8 \mathrm{~kg})(P<0.001)$. The median age (years) of the extreme brachycephalic breed types (Bulldog 2.0, French Bulldog 1.2, Pug 2.0) was younger than for the moderate and non-brachycephalic breed types (Yorkshire Terrier 5.3, Border Terrier 5.2, West Highland White Terrier 6.9) $(P<0.001)$. The median time (years) contributed to the study (period from the first to the final patient record) was shorter for the extreme brachycephalic breed types (Bulldog 0.2, French Bulldog 0.2, Pug 0.2) than for the moderate and nonbrachycephalic breed types (Yorkshire Terrier 0.8, Border Terrier 1.0, West Highland White Terrier 1.0) $(P<0.001)$ (Table 2).

During the study period, 83 (6.9\%) dogs died, with 67 (80.7 \%) of these deaths involving euthanasia. The median (IQR) age at death among the study dogs overall was $12.0(9.5-14.2)$ years. The mortality of extreme brachycephalic dogs $(24,4.0 \%)$ during the study period was lower than for moderate and non-brachycephalic dogs (59 deaths, $9.8 \%)(P<0.001)$. The median (IQR) age at death for extreme brachycephalic dogs (8.6, 2.4-10.8) was younger than for moderate and non-brachycephalic dogs $(12.7,11.1-15.0)(P<0.001)$. A higher proportion of deaths were ascribed to URT disorders for extreme brachycephalic breed types (4 cases, $16.7 \%$ ) compared with moderate and non-brachycephalic breed types $(0,0.0 \%)$ $(P=0.001)$.

The prevalence of URT disorders among the study dogs overall was $15.8 \%$ (95 \% CI: 13.2-18.4). The prevalence of URT disorders was higher in extreme brachycephalic dogs $(22.0 \%, 95 \%$ CI: $18.0-26.0)$ than in the moderate and non-brachycephalic group $(9.7 \%, 95 \%$ CI: 7.1-12.3) $(P<0.001)$. Compared with the moderate and non-brachycephalic dogs, the extreme brachycephalic dogs had higher prevalence of disorders affecting the nares/nasal cavity $(P<0.001)$, hard and soft palate $(P<0.001)$, larynx $(P=0.033)$, BOAS $(P<0.001)$ and multi-site URT $(P<0.001)$ (Table 3$)$.

The prevalence of having at least one URT disorder varied significantly between the individual breed types: Bulldog 19.5 \%, French Bulldog 20.0 \%, Pug $26.5 \%$, Yorkshire Terrier $13.0 \%$, Border Terrier $9.0 \%$ and West Highland White Terrier $7.0 \%(P<0.001)$. Individual breed types also varied significantly for some locations of URT disorders: nares/nasal cavity $(P<0.001)$, hard and soft palate $(P<0.001)$, BOAS $(P<0.001)$ and multisite URT $(P<0.001)$ (Table 4$)$.

Using univariable logistic regression modelling, six variables showed liberally significant $(P<0.20)$ associations with an outcome of having at least one URT disorder diagnosed: extreme brachycephalic/moderate and

Table 3 Comparative prevalence values (\%) for upper respiratory tract (URT) disorders recorded in 600 extreme brachycephalic (Bulldog, French Bulldog, Pug) and 600 other dogs (moderate brachycephalic: Yorkshire Terrier, non-brachycephalic: Border Terrier, West Highland White Terrier) attending primary-care veterinary practices in England (200 dogs of each breed type)

\begin{tabular}{|c|c|c|c|c|}
\hline Variable & $\begin{array}{l}\text { Extreme brachycephalic } \\
(n=600) \text { No. (\%) }\end{array}$ & $\begin{array}{l}\text { Moderate and non-brachycephalic } \\
(n=600) \text { No. }(\%)\end{array}$ & Overall & $P$-value \\
\hline Proportion of dogs with at least one URT disorder & $132(22.0)$ & $58(9.7)$ & $190(15.8)$ & $<0.001$ \\
\hline \multicolumn{5}{|c|}{ Proportion of dogs with a URT disorder affecting this anatomic site } \\
\hline Nares/Nasal Cavity & $68(11.3)$ & $23(3.8)$ & $91(7.6)$ & $<0.001$ \\
\hline Hard and soft palate & $19(3.2)$ & $0(0.0)$ & $19(1.6)$ & $<0.001$ \\
\hline Pharynx & $8(1.3)$ & $4(0.7)$ & $12(1.0)$ & 0.246 \\
\hline Tonsil & $3(0.5)$ & $1(0.2)$ & $4(0.3)$ & 0.317 \\
\hline Larynx & $7(1.2)$ & $1(0.2)$ & $8(0.7)$ & 0.033 \\
\hline Trachea & $30(5.0)$ & $38(6.3)$ & $68(5.7)$ & 0.318 \\
\hline BOAS (brachycephalic obstructive airway syndrome) & $21(3.5)$ & $0(0.0)$ & $21(1.8)$ & $<0.001$ \\
\hline Multi-site URT & $68(11.3)$ & $11(1.8)$ & $79(6.6)$ & $<0.001$ \\
\hline
\end{tabular}


Table 4 Comparative prevalence values (\%) for upper respiratory tract (URT) disorders recorded in three extreme brachycephalic breed types (Bulldog, French Bulldog, Pug) and three other breed types (moderate brachycephalic: Yorkshire Terrier, non-brachycephalic: Border Terrier, West Highland White Terrier) of dog attending primary-care veterinary practices in England (200 dogs of each breed type)

\begin{tabular}{|c|c|c|c|c|c|c|c|}
\hline Variable & $\begin{array}{l}\text { Bulldog }(n=200) \\
\text { No. }(\%)\end{array}$ & $\begin{array}{l}\text { French bulldog } \\
(n=200) \text { No. }(\%)\end{array}$ & $\begin{array}{l}\text { Pug }(n=200) \\
\text { No. }(\%)\end{array}$ & $\begin{array}{l}\text { Border terrier } \\
(n=200) \text { No. }(\%)\end{array}$ & $\begin{array}{l}\text { West highland } \\
\text { white terrier } \\
(n=200) \text { No. (\%) }\end{array}$ & $\begin{array}{l}\text { Yorkshire terrier } \\
(n=200) \text { No. }(\%)\end{array}$ & $\begin{array}{l}P- \\
\text { value }\end{array}$ \\
\hline $\begin{array}{l}\text { Proportion of dogs with at least one } \\
\text { URT disorder }\end{array}$ & $39(19.5)$ & $40(20.0)$ & $53(26.5)$ & $18(9.0)$ & $14(7.0)$ & $26(13.0)$ & $<0.001$ \\
\hline \multicolumn{8}{|l|}{$\begin{array}{l}\text { Proportion of dogs with a URT } \\
\text { disorder affecting this anatomic site }\end{array}$} \\
\hline Nares/Nasal Cavity & $15(7.5)$ & $24(12.0)$ & $29(14.5)$ & $8(4.0)$ & $7(3.5)$ & $8(4.0)$ & $<0.001$ \\
\hline Hard and soft palate & $5(2.5)$ & $6(3.0)$ & $8(4.0)$ & $0(0.0)$ & $0(0.0)$ & $0(0.0)$ & 0.001 \\
\hline Pharynx & $2(1.0)$ & $2(1.0)$ & $4(2.0)$ & $1(0.5)$ & $2(1.0)$ & $1(0.5)$ & 0.695 \\
\hline Tonsil & $0(0.0)$ & $3(1.5)$ & $0(0.0)$ & $0(0.0)$ & $0(0.0)$ & $1(0.5)$ & 0.051 \\
\hline Larynx & $1(0.5)$ & $3(1.5)$ & $3(1.5)$ & $1(0.5)$ & $0(0.0)$ & $0(0.0)$ & 0.217 \\
\hline Trachea & $7(3.5)$ & $12(6.0)$ & $11(5.5)$ & $10(5.0)$ & $8(4.0)$ & $20(10.0)$ & 0.074 \\
\hline $\begin{array}{l}\text { BOAS (brachycephalic obstructive } \\
\text { airway syndrome) }\end{array}$ & $5(2.5)$ & $3(1.5)$ & $13(6.5)$ & $0(0.0)$ & $0(0.0)$ & $0(0.0)$ & $<0.001$ \\
\hline Multi-site URT & $21(10.5)$ & $21(10.5)$ & $26(13.0)$ & $5(2.5)$ & $2(1.0)$ & $4(2.0)$ & $<0.001$ \\
\hline
\end{tabular}

non-brachycephalic status, breed type, age category, insurance status, extreme brachycephalic/moderate and non-brachycephalic weight tertiles and breed type weight tertiles. In order to separately evaluate extreme brachycephalic/moderate and non-brachycephalic status and breed type as risk factors of main interest for URT disorder diagnosis, two multivariable logistic regression models were built that each additionally evaluated the age category and insurance status of individual dogs but used the appropriate bodyweight tertile variable. Both final models comprised three risk factors: insurance status and weight tertiles along with either extreme brachycephalic/moderate and nonbrachycephalic status or breed type. No biologicallysignificant interactions were identified in either final model. Both final non-clustered models showed acceptable model-fit (Hosmer-Lemeshow test statistics: brachycephalic status model $P=0.493$ and individual breed type model $P=0.553$ ) and moderate discrimination (area under the ROC curve: brachycephalic status model 0.684 and breed type model 0.698). Both final models were improved by inclusion of the clinic attended as a random effect (brachycephalic status model $P=0.043 /$ rho $=0.03$ and breed type model $P=$ $0.034 /$ rho $=0.03$ ), indicating that $3 \%$ of variation for each models was accounted for by the clinic attended. After accounting for the effects of the other variables evaluated, the extreme brachycephalic dogs were associated with 3.5 times (95 \% CI: 2.4-5.0, $P<0.001$ ) the odds of diagnosis with at least one URT disorder compared with the moderate and non-brachycephalic group (Table 5). Following adjustment, individual breed type categorisation was also significantly associated with diagnosis with at least one URT disorder $(P<0.001)$. The Bulldog (odds ratio (OR) 4.0, $95 \%$ CI 2.1-7.9, $P<0.001$ ), French Bulldog (OR 5.1, 95 \% CI 2.6-10.2, P<0.001), Pug (OR 6.9, 95 \% CI 3.6-13.3, $P<0.001$ ) and Yorkshire Terrier (OR 2.2, $95 \%$ CI 1.1-4.5, $P=0.026$ ) had higher odds of diagnosis with at least one URT disorder compared with the West Highland

Table 5 Final multivariable logistic regression model with brachycephalic status as the factor of primary interest for association with diagnosis with at least one upper respiratory tract disorder in dogs attending primary-care veterinary practices in England

\begin{tabular}{|c|c|c|c|c|}
\hline Variable & Category & Odds ratio & $95 \% \mathrm{Cl}$ & $P$-value ${ }^{a}$ \\
\hline \multirow[t]{2}{*}{ Brachycephalic status } & Moderate and non-brachycephalic & Base & & \\
\hline & Extreme brachycephalic & 3.5 & $2.4-5.0$ & $<0.001$ \\
\hline \multirow[t]{4}{*}{ Bodyweight tertiles within groups } & High & Base & & $(<0.001)$ \\
\hline & Mid & 1.1 & $0.7-1.9$ & 0.612 \\
\hline & Low & 1.8 & $1.1-2.9$ & 0.016 \\
\hline & No recorded bodyweight & 0.6 & $0.4-1.0$ & 0.057 \\
\hline \multirow[t]{2}{*}{ Insurance } & Non-insured & Base & & \\
\hline & Insured & 1.8 & $1.2-2.7$ & 0.003 \\
\hline
\end{tabular}

${ }^{\text {a }}$ For variables with $>2$ categories, the overall $P$-value is shown in brackets 
Table 6 Final multivariable logistic regression model evaluating risk factors for association with diagnosis of at least one upper respiratory tract disorder in dogs attending primary-care veterinary practices in England. Breed type was the factor of primary interest

\begin{tabular}{|c|c|c|c|c|}
\hline Variable & Category & Odds ratio & $95 \% \mathrm{Cl}$ & $P$-value ${ }^{a}$ \\
\hline \multirow[t]{6}{*}{ Breed type } & West Highland White & Base & & $(<0.001)$ \\
\hline & Border Terrier & 1.4 & $0.6-2.9$ & 0.409 \\
\hline & Yorkshire Terrier & 2.2 & $1.1-4.5$ & 0.026 \\
\hline & Bulldog & 4.0 & $2.1-7.9$ & $<0.001$ \\
\hline & French Bulldog & 5.1 & $2.6-10.2$ & $<0.001$ \\
\hline & Pug & 6.9 & $3.6-13.3$ & $<0.001$ \\
\hline \multirow{4}{*}{$\begin{array}{l}\text { Bodyweight categories within extreme brachycephalic } \\
\text { and moderate and non-brachycephalic categories }\end{array}$} & High & Base & & $(<0.001)$ \\
\hline & Mid & 1.3 & $0.8-2.1$ & 0.324 \\
\hline & Low & 1.8 & $1.1-2.9$ & 0.020 \\
\hline & No recorded bodyweight & 0.6 & $0.4-1.1$ & 0.079 \\
\hline \multirow[t]{2}{*}{ Insurance } & Non-insured & Base & & \\
\hline & Insured & 1.9 & $1.3-2.8$ & 0.002 \\
\hline
\end{tabular}

${ }^{\mathrm{a}}$ For variables with $>2$ categories, the overall $P$-value is shown in brackets

White Terrier (Table 6). In both the extreme brachycephalic/moderate and non-brachycephalic and the breed type models, being insured and being in the lower bodyweight tertile had increased odds of diagnosis with at least one URT disorder.

\section{Discussion}

This study used clinical data on randomly selected dogs of extreme brachycephalic and of moderate and nonbrachycephalic breed types attending a group of veterinary practices in England to report and compare the prevalence of URT disorders diagnosed. Upper respiratory tract disorders were a frequent diagnosis overall $(15.8 \%)$ but were more common in extreme brachycephalic dogs (22.0\%) than the moderate and non-brachycephalic group (9.7\%). The most prevalent anatomic sites affected by URT disorders were the nares/nasal cavity (7.6\%), multi-site URT (6.6 \%) and trachea (5.7 \%). The prevalence of dogs with at least one URT disorder diagnosed varied widely between the breed types evaluated, ranging from $26.5 \%$ of Pugs to $7.0 \%$ of WHWTs affected. These results support a predisposition to URT disorders in extreme brachycephalic breed types but also identify a substantial disease burden for URT disorders in moderate and non-brachycephalic breed types.

Brachycephaly has been reported to reduce general quality of life by decreasing exercise tolerance, increasing recovery time after physical exercise and from associations with a variety of sleep problems and breathlessness $[16,22]$. This study specifically aimed to contribute to the evidence base on links between skull morphology and URT health in dogs. The current study grouped breed types into three categories (extreme, moderate or non brachycephalic) based on skull morphology in consistency with protocols used in many other studies [11, 23]. However, the genetic basis for planned selections for skull distortions in modern dog breeds is highly complex $[24,25]$ and, in the future, it may become more useful to describe the metrics of skull morphology as a continuum across the modern domestic dog breeds [14]. The Bulldog, French Bulldog and Pug are characteristically of extreme brachycephalic conformation in terms of their short "pushed-in" faces, under-bite, and widely placed, shallow orbits [26] and are commonly reported as showing predisposition to URT disorders [27, 28], although reliable prevalence data are limited. The current study aimed to fill this data gap by reporting and comparing the prevalence of URT disorders between three extreme brachycephalic and three moderate and non-brachycephalic breed types. The moderate (Yorkshire Terrier) and nonbrachycephalic (Border Terrier and WHWT) comparator breed types were selected because they were small-to-medium sized breed types that are commonly owned in the general population, thus providing results that are relevant to large numbers of dogs as well as avoiding excessive bodyweight disparity between the groups [16].

The current study identified that URT disorders represented a relatively common diagnosis among the overall study population of breed types included, with almost one in six dogs having at least one URT disorder diagnosed. Despite a high interest in the welfare impact of URT disorders in certain breeds, there are limited prevalence data available on these disorders in dogs to inform evidence-based reforms [20, 29]. A US study of practice- 
attending dogs failed to identify any URT disorders among the 29 most common disorders diagnosed [30] but this may reflect the wide spread of specific diagnostic terms used for URT disorders that may result in relatively low prevalence values for each individual disorder. A study of overall disorder prevalence among practiceattending dogs in England reported no specific URT disorders among the twenty most common specific disorders as diagnosed, but $5.7 \%$ of dogs were shown to have at least one URT disorder after grouping the disparate URT disorders into a single disorder category [31].

The results of the current study support the hypothesis that extreme brachycephaly is a strong risk factor for URT disorders in dogs. After accounting for measured differences between the groups, extreme brachycephalic dogs had 3.5 times the odds of being diagnosed with at least one URT disorder compared with moderate and non-brachycephalic dogs. This finding concurs with the majority of other evidence that identifies brachycephaly as a strong risk factor for URT disorders. Using a wide variety of information resources, a review of inherited disorders related to breed standards for the 50 most common dog breeds reported that brachycephalic breeds had significantly more respiratory disorders than mesaticephalic and dolichocephalic breeds and concluded that skull shape affected the respiratory disorders to which breeds were predisposed [23]. Brachycephaly across modern breeds of dog is regularly accompanied by a spectrum of bony and soft tissue differences compared with their original progenitor wolf ancestors. Many modern brachycephalic breeds are characterised by a shortening of the muzzle bones of the skull without an equivalent reduction in the volume of the associated nasopharyneal soft tissues, requiring increased negative pressure during inspiration to overcome obstructed and turbulent airflow $[25,32]$ that leads to stretching and inflammation of the URT tissues and predisposes to clinical URT disorders [27]. Miniaturisation of breeds in the quest for more extreme conformations can increase the relative disparity between the bony and the soft tissues of the head and lead to even greater health impacts [33].

In humans, obesity and pharyngeal abnormalities have been linked with pharyngeal collapsibility and URT problems [34]. Human obesity leads to increased soft tissue within the fixed bony structures defined by the maxilla, mandible and vertebra and results in a reduced airway lumen [34]. However, evidence for a similar association between higher bodyweight and URT disorders in dogs is less clear. In the current study, lower relative bodyweight was associated with increased odds of URT disorder. After accounting for other factors, dogs in the bottom tertile of bodyweight had almost twice the odds of an URT disorder compared with dogs in the top tertile. A study of 73 dogs presented for BOAS surgery showed no trend for affected animals to weigh more than animals of the same breed presented for other problems [35]. Bodyweight is a complex interaction of many factors and does not necessarily accurately reflect obesity, even within breeds [36]. Future studies that collect body condition score information are required to specifically evaluate the role of obesity in URT disorders.

The current study found no evidence of a sex predisposition to URT disorders in dogs. Previous studies have similarly reported inconsistent sex findings. A study of 90 dogs affected with BOAS indicated a male predisposition in [27] whereas a study of 73 dogs affected with BOAS indicated a female predisposition [35]. These two studies focused purely on dogs referred for BOAS treatment and were based on smaller case numbers and thus may be more biased than the current study which included records from 600 dogs attending primary-care veterinary practices for any reason.

There were some limitations to this study. The prevalence values reported for the extreme brachycephalic and moderate and non-brachycephalic dogs relate to the specific breed types evaluated and do not necessarily apply to all other breed types of comparable skull conformation. Prevalence results for other breed types may differ by the degree of brachycephaly, bodyweight, age structures and other factors. The study dogs attended a single large veterinary partnership group and were mostly located in central and south-eastern England and thus may be less representative of cases seen in other parts of the country or at other practice types. Studies based on reviews of medical records of animals may under-estimate the true disease burden by predominantly including more severely affected animals that warrant veterinary management but with less success at identifying less severely affected cases that are less likely to be clinically presented [16].

Although the current study identified a higher prevalence of URT disorders in extreme brachycephalic compared with moderate and non-brachycephalic dogs, these results may even have under-estimated the true disparity between these groups. A normalisation phenomenon is increasingly recognised that describes the acceptance as 'normal' of some common chronic and highly breedassociated conditions [35]. The owners of over half of dogs diagnosed with BOAS at a referral centre stated that their dog did not have breathing problems [18]. This normalisation phenomenon may blind owners and veterinarians to URT disorders in commonly affected breeds and lead to under-reporting and under-diagnosis. In recent years, the Pug and French Bulldog in particular have experienced a phenomenal surge in popularity [12], partly explaining why the extreme brachycephalic breed types were younger than the moderate and non-brachycephalic breed types in the current study. The clinical signs of URT disorders associated with brachycephaly are reported to exacerbate as 
dogs age $[16,37]$, suggesting that the difference in URT disorder prevalence between the extreme brachycephalic breed types and their moderate and non-brachycephalic counterparts might have been even greater if the two groups were age-matched.

\section{Conclusions}

This study reports that URT disorders are relatively commonly diagnosed across Bulldog, French Bulldog, Pug, Border Terrier, WHWT and Yorkshire Terrier dogs attending primary-care veterinary practices in England. The three evaluated breed types of extreme brachycephalic conformation (Bulldog, French Bulldog and Pug) were relatively short-lived and predisposed to URT disorders compared with three other small-to-medium sized breed types that are commonly owned (moderate brachycephalic: Yorkshire Terrier and non-brachycephalic: Border Terrier and WHWT). These findings expand the evidence base available to practitioners to aid clinical decision-making and should assist reforms to improve canine breed welfare.

\section{Methods}

The VetCompass Animal Surveillance programme collates de-identified electronic patient record (EPR) data from selected primary-care veterinary practices in the UK for epidemiological research [31]. Collaborating practices were selected by their willingness to participate and their recording of clinical data within an appropriately configured practice management system (PMS). Practitioners recorded summary diagnosis terms from an embedded VeNom Code list during episodes of care. Information collected related generally to the owned dog population and included patient demographic (species, breed type, date of birth, sex, neuter status, insurance status and weight) and clinical information (free-form text clinical notes, summary diagnosis terms, treatment and deceased status with relevant dates) data fields. Integrated clinical queries were used to extract EPR data from PMSs for upload to a secure VetCompass structured query language database [38]. Ethical approval of the project was granted by the RVC Ethics and Welfare Committee (reference number 20101076 h).

The sampling frame for the current study included all dogs that attended any practice within the Medivet Veterinary Partnership from September 1, 2009 until March 2, 2014 with at least one EPR describing a clinical note, bodyweight or treatment dispensed recorded in the VetCompass database. The Medivet Veterinary Partnership is a large group of integrated veterinary practices covering central and south-eastern England [39]. A historical cohort study design was used. Study animals were selected from the overall sampling frame using stratified random sampling. Two hundred dogs were randomly selected from each of six breed types of interest (Bulldog, French Bulldog, Pug, Yorkshire Terrier, Border Terrier and West Highland White Terrier (WHWT)). Randomisation used a web-based random number generator [40]. The URT was defined to include those sections of the respiratory system extending from the external nares to the distal trachea and also to include the oropharynx [41]. A URT disorder was defined as any disorder recorded in the veterinary EPR that related primarily to any part of the URT. The 'clinical note' and 'summary diagnosis' fields for each selected dog were manually reviewed to extract data on all URT disorders and mortality events recorded during the study period. Sample size calculations estimated a cohort study with 544 exposed and 544 non-exposed dogs would have $80 \%$ power to detect a risk factor with an odds ratio of 3.0 or greater having a $1.5 \%$ prevalence in the non-exposed animals (two-sided $\alpha=0.05$ ) [42].

Bulldog, French Bulldog and Pug breed types were classified as extreme brachycephalic, the Yorkshire Terrier breed type was classified as moderate brachycephalic and the Border Terrier and West Highland White Terrier breed types were classified as nonbrachycephalic [10]. The neuter and age values at the final EPR were used. Sex and neuter were combined to create a sex/neuter variable with four categories: female entire, female neutered, male entire and male neutered. Insurance status described whether a dog was insured at any point during the study period. Age (in years) at the final EPR was reported as a continuous variable and also categorised into five groups $(<3.0,3.0-5.9,6.0-8.9,9.0-11.9, \geq 12.0)$. The maximum bodyweight values recorded for dogs aged 9 months and above were categorised into 4 groups $(0.0-9.9 \mathrm{~kg}$, $10.0-19.9 \mathrm{~kg}, 20.0 \mathrm{~kg}$ and above, no weight recorded). Relative bodyweight values were created by grouping bodyweights into tertiles within the extreme brachycephalic/moderate and non-brachycephalic groups and for individual breed types. The time contributed to the study for each dog was defined as the period between the first and the final clinical record. The recorded URT disorders were categorised according to the affected anatomic site as described in the clinical notes (nares/nasal cavity, larynx, palate, pharynx, tonsil and trachea) with two additional categories added to cover disorders that were not restricted to a single anatomic area (BOAS and multi-site URT).

Following data checking and cleaning in Excel (Microsoft Office Excel 2007, Microsoft Corp.), statistical analyses were conducted using Stata Version 11.2 (Stata Corporation). Prevalence values with $95 \%$ confidence intervals (95\% CI) were reported separately for the overall study population of dogs, for extreme 
brachycephalic and moderate and non-brachycephalic groups, and for each breed type. The $95 \%$ CI estimates were derived from standard errors based on approximation to the normal distribution for disorders with $\geq 10$ events [43]. Descriptive statistics characterised the sex/neuter, insurance status, age, weight and time contributed to the study for the six breed types under investigation, extreme brachycephalic and moderate and non-brachycephalic status, and the study dogs overall. Categorical demographic characteristics and prevalence values were compared between individual breed types and between extreme brachycephalic and moderate and non-brachycephalic groups using the chi-square test or Fishers exact test as appropriate while age, bodyweight and time in study were compared using the Wilcoxon rank sum test or the Kruskal Wallis test, as appropriate [43].

Binary logistic regression modelling was used to evaluate brachycephalic and individual breed type statuses separately as risk factors for having at least one URT disorder, taking account of sex/neuter, weight tertile and insured status as possible confounding variables. Factors with liberal associations in univariable modelling $(P<0.2)$ were taken forward for multivariable evaluation. Model development used backwards stepwise elimination. Clinic attended was entered as a random effect and pair-wise interaction effects were evaluated for the final model variables [44]. The Hosmer-Lemeshow test statistic and the area under the ROC curve were used to evaluate model fit (non-random effect model) [44]. Statistical significance was set at $P<0.05$.

\section{Abbreviations \\ BOAS: Brachycephalic obstructive airway syndrome; Cl: Confidence interval; EPR: Electronic patient record; OR: Odds ratio; PMS: Practice management system; URT: Upper respiratory tract; WHWT: West Highland White Terrier.}

\section{Competing interests}

Dan O'Neill is currently funded to work at the Royal Veterinary College by a Kennel Club Charitable Trust award. The work described in the current paper was carried out prior to taking on this role. All other authors declare no competing interests.

\section{Authors' contributions}

DON: project conception, project design, data collection, data extraction, analysis, drafting the manuscript. CJ: project conception, project design, data extraction, analysis, drafting the manuscript. JHG: project conception, project design, drafting the manuscript. DBC: project design, data collection, drafting the manuscript. PDM: project design, data collection, drafting the manuscript. PCT: data collection, analysis, drafting the manuscript. DCB: project design, data collection, analysis, drafting the manuscript. All authors read and approved the final manuscript

\section{Acknowledgements}

We are grateful to Dogs Trust for supporting this work. Thanks to Peter Dron (RVC) and Noel Kennedy (RVC) for database, software and programming development associated with the VetCompass Programme. We are particularly grateful to the veterinary practitioners and clients of the Medivet Veterinary Group and other independent practices who participate in VetCompass and share de-identified clinical data on their animals.

\section{Author details}

'The Royal Veterinary College, Hawkshead Lane, North Mymms, HatfieldHerts AL9 7TA, UK. ${ }^{2}$ School of Agriculture, Food and Rural Development, Newcastle University, Newcastle upon Tyne, NE1 7RU, UK. ${ }^{3}$ Gunn Building (B19), Faculty of Veterinary Science, The University of Sydney, Sydney, NSW 2006, Australia.

Received: 16 February 2015 Accepted: 7 July 2015

Published online: 14 July 2015

\section{References}

1. Irion DN, Schaffer AL, Famula TR, Eggleston ML, Hughes SS, Pedersen NC. Analysis of genetic variation in 28 dog breed populations with 100 microsatellite markers. J Heredity. 2003;94(1):81-7. doi:10.1093/jhered/ esg004.

2. Clutton-Brock J. Dog. In: Mason IL, editor. Evolution of domesticated animals. London: Longman; 1984. p. 198-211.

3. Sampson J, Binns MM. The Kennel Club and the early history of dog shows and breed clubs. In: Ostrander E, editor. The Dog and Its Genome. USA: CSHL Press; 2007. p. 19-30.

4. Parker HG, Kim LV, Sutter NB, Carlson S, Lorentzen TD, Malek TB, et al. Genetic structure of the purebred domestic dog. Science. 2004:304(5674):1160-4.

5. Alderton D, Morgan T. Dogs. 1st ed. London: Dorling Kindersley; 1993.

6. Neff MW, Rine J. A fetching model organism. Cell. 2006;124(2):229-31. doi:10.1016/j.cell.2006.01.008.

7. Lindblad-Toh K. Genome sequence, comparative analysis and haplotype structure of the domestic dog. Nature. 2005;438(7069):803-19.

8. Drake $A G$, Klingenberg CP. Large scale diversification of skull shape in domestic dogs: disparity and modularity. Am Nat. 2010;175(3):289-301.

9. Schmidt MJ, Neumann AC, Amort KH, Failing K, Kramer M. Cephalometric measurements and determination of general skull type of Cavalier King Charles Spaniels. Vet Radiol Ultrasound. 2011;52(4):436-40. doi:10.1111/ j.1740-8261.2011.01825.x

10. Georgevsky D, Carrasco JJ, Valenzuela M, McGreevy PD. Domestic dog skull diversity across breeds, breed groupings, and genetic clusters. J Vet Behav: Clin Appl Res. 2014;9(5):228-34. http://dx.doi.org/10.1016/j.jveb.2014.04.007

11. Hussein AK, Sullivan M, Penderis J. Effect of brachycephalic, mesaticephalic, and dolichocephalic head conformations on olfactory bulb angle and orientation in dogs as determined by use of in vivo magnetic resonance imaging. Am J Vet Res. 2012;73(7):946-51. doi:10.2460/ajvr.73.7.946.

12. The Kennel Club. Breed registration statistics. The Kennel Club Limited. 2014 http://www.thekennelclub.org.uk/registration/breed-registration-statistics/. Accessed January 192015.

13. Emmerson T. Brachycephalic obstructive airway syndrome: a growing problem. J Small Anim Pract. 2014;55(11):543-4. doi:10.1111/jsap.12286.

14. McGreevy PD, Georgevsky D, Carrasco J, Valenzuela M, Duffy DL, Serpell JA. Dog behavior co-varies with height, bodyweight and skull shape. PLoS One. 2013:8(12), e80529. doi:10.1371/journal.pone.0080529.

15. Aron D, Crowe D. Upper airway obstruction. General principles and selected conditions in the dog and cat. Vet Clin North Am Small Anim Pract. 1985;15(5):891-917

16. Roedler FS, Pohl S, Oechtering GU. How does severe brachycephaly affect dog's lives? Results of a structured preoperative owner questionnaire. Vet J. 2013;198(3):606-10. http://dx.doi.org/10.1016/j.tvjl.2013.09.009.

17. Koch DA, Arnold S, Hubler M, Montavon P. Brachycephalic syndrome in dogs. Compend Contin Educ Pract Vet - North Am Edition. 2003;25(1):48-55.

18. Packer RMA, Hendricks A, Burn CC. Do dog owners perceive the clinical signs related to conformational inherited disorders as 'normal' for the breed? A potential constraint to improving canine welfare. Anim Welfare. 2012;21(Supplement 1):81-93. doi:10.7120/ $096272812 \times 13345905673809$.

19. Anon. Balancing pedigree dog breed standards and animal welfare - is it possible? Vet Rec. 2009;164(16):481-2.

20. Bateson P. Independent inquiry into dog breeding. Cambridge: University of Cambridge; 2010.

21. O'Neill DG, Church DB, McGreevy PD, Thomson PC, Brodbelt DC. Longevity and mortality of owned dogs in England. Vet J. 2013;198(3):638-43. doi:10.1016/j.tvjl.2013.09.020.

22. Beausoleil NJ, Mellor DJ. Introducing breathlessness as a significant animal welfare issue. N Z Vet J. 2015;63(1):44-51. doi:10.1080/00480169.2014.940410. 
23. Asher L, Diesel G, Summers JF, McGreevy PD, Collins LM. Inherited defects in pedigree dogs. Part 1: disorders related to breed standards. Vet J. 2009;182(3):402-11. doi:10.1016/j.tvjl.2009.08.033

24. Schoenebeck JJ, Hutchinson SA, Byers A, Beale HC, Carrington B, Faden DL, et al. Variation of BMP3 contributes to dog breed skull diversity. PLoS Genet. 2012;8(8), e1002849. doi:10.1371/journal.pgen.1002849.

25. Bannasch D, Young A, Myers J, Truvé K, Dickinson P, Gregg J, et al. Localization of canine brachycephaly using an across breed mapping approach. PLoS One. 2010;5(3), e9632. doi:10.1371/journal.pone.0009632.

26. Schoenebeck JJ, Ostrander EA. The genetics of canine skull shape variation. Genetics. 2013;193(2):317-25. doi:10.1534/genetics.112.145284.

27. Fasanella FJ, Shivley JM, Wardlaw JL, Givaruangsawat S. Brachycephalic airway obstructive syndrome in dogs: 90 cases (1991-2008). J Am Vet Med Assoc. 2010;237(9):1048-51. doi:10.2460/javma.237.9.1048.

28. De Lorenzi D, Bertoncello D, Drigo M. Bronchial abnormalities found in a consecutive series of 40 brachycephalic dogs. J Am Vet Med Assoc. 2009;235(7):835-40. doi:10.2460/javma.235.7.835.

29. Collins LM, Asher L, Summers J, McGreevy P. Getting priorities straight: risk assessment and decision-making in the improvement of inherited disorders in pedigree dogs. Vet J. 2011;189(2):147-54. doi:10.1016/j.tvjl.2011.06.012.

30. Lund EM, Armstrong PJ, Kirk CA, Kolar LM, Klausner JS. Health status and population characteristics of dogs and cats examined at private veterinary practices in the United States. J Am Vet Med Assoc. 1999;214(9):1336-41.

31. O'Neill DG, Church DB, McGreevy PD, Thomson PC, Brodbelt DC. Prevalence of disorders recorded in dogs attending primary-care veterinary practices in England. PLoS One. 2014;9(3):1-16. doi:10.1371/journal.pone.0090501.

32. Bernaerts F, Talavera J, Leemans J, Hamaide A, Claeys S, Kirschvink N, et al. Description of original endoscopic findings and respiratory functional assessment using barometric whole-body plethysmography in dogs suffering from brachycephalic airway obstruction syndrome. Vet J. 2010;183(1):95-102. http://dx.doi.org/10.1016/j.tvjl.2008.09.009.

33. Rusbridge C. Chiari-like malformation and syringomyelia. ECJAP. 2013;23(3):70-89.

34. Isono S. Contribution of obesity and craniofacial abnormalities to pharyngeal collapsibility in patients with obstructive sleep apnea. Sleep Biol Rhythms. 2004;2(1):17-21. doi:10.1111/j.1479-8425.2003.00081.x.

35. Torrez CV, Hunt GB. Results of surgical correction of abnormalities associated with brachycephalic airway obstruction syndrome in dogs in Australia. J Small Anim Pract. 2006;47(3):150-4. doi:10.1111/j.17485827.2006.00059.x.

36. German AJ. The growing problem of obesity in dogs and cats. J Nutr. 2006;136(7):1940S-6.

37. Knecht CD. Upper airway obstruction in brachycephalic dogs. Compend Contin Educ Practicing Vet. 1979;1:25-31.

38. O'Neill DG, Elliott J, Church DB, McGreevy PD, Thomson PC, Brodbelt DC. Chronic kidney disease in dogs in UK veterinary practices: prevalence, risk factors, and survival. J Vet Intern Med. 2013;27(4):814-21. doi:10.1111/ jvim. 12090.

39. Medivet. Medivet: the veterinary partnership. Medivet Partnership LLP. 2014. http://www.medivet.co.uk/. Accessed January 142014.

40. Haahr M. RANDOM. ORG: True random number service. In: http:// www.random.org/. TSDA. 2012. http://www.random.org/. Accessed March 17th 2012.

41. Fonfara S, de la Heras AL, German AJ, Blackwood L, Dukes-McEwan J, Noble $P J$, et al. Underlying diseases in dogs referred to a veterinary teaching hospital because of dyspnea: 229 cases (2003-2007). J Am Vet Med Assoc. 2011:239(9):1219-24. doi:10.2460/javma.239.9.1219.

42. Epi Info 7 CDC. Centers for Disease Control and Prevention (US): Introducing Epi Info 7. CDC, Atlanta, Georgia. 2012. http://wwwn.cdc.gov/ Epiinfo/7/index.htm. Accessed May 8th 2012

43. Kirkwood BR, Sterne JAC. Essential Medical Statistics. 2nd ed. Oxford: Blackwell Science; 2003.

44. Dohoo I, Martin W, Stryhn H. Veterinary Epidemiologic Research. 2nd ed. Charlottetown, Canada: VER Inc; 2009.

\section{Submit your next manuscript to BioMed Central and take full advantage of:}

- Convenient online submission

- Thorough peer review

- No space constraints or color figure charges

- Immediate publication on acceptance

- Inclusion in PubMed, CAS, Scopus and Google Scholar

- Research which is freely available for redistribution

Submit your manuscript at www.biomedcentral.com/submit 